\title{
Téoros
}

Revue de recherche en tourisme

\section{Le concept de cluster est-il soluble dans le tourisme ?}

\section{Cécile Clergeau et Philippe Violier}

Volume 31, numéro 2, 2012

URI : https://id.erudit.org/iderudit/1020772ar

DOI : https://doi.org/10.7202/1020772ar

Aller au sommaire du numéro

\section{Éditeur(s)}

Université du Québec à Montréal

\section{ISSN}

0712-8657 (imprimé)

1923-2705 (numérique)

Découvrir la revue

\section{Citer cet article}

Clergeau, C. \& Violier, P. (2012). Le concept de cluster est-il soluble dans le tourisme? Téoros, 31(2), 60-71. https://doi.org/10.7202/1020772ar

\section{Résumé de l'article}

Dans les approches scientifiques, le concept de cluster (grappe) met en évidence, d'une part, le rôle des proximités organisationnelles, construites à partir des proximités spatiales dans la dynamique de compétitivité des destinations touristiques. D'autre part, des clusters tourisme se développent partout dans le monde. Or, le concept de cluster, tel que proposé par Porter (1998), émane de l'économie industrielle.

L'enjeu de cet article consiste donc à montrer que le simple transfert d'un outil scientifique élaboré dans le contexte industriel au management des destinations touristiques pose problème. Il doit être enrichi pour comprendre les dynamiques de développement touristique local et acquérir plus d'efficacité opérationnelle. Trois axes de réflexion théorique, mais inspirés par des études de cas rencontrées dans différents travaux de recherche, sont proposés pour interroger les analyses des clusters tourisme. Il convient en effet de prendre en compte trois dimensions spécifiques qui seront abordées à partir du processus de coproduction inhérent à l'activité touristique, des dynamiques de proximités singulières dans ce champ du tourisme et des caractéristiques de l'encastrement des activités touristiques dans le territoire. Enfin, les auteurs proposent un modèle de cluster plus adapté aux destinations touristiques.
Ce document est protégé par la loi sur le droit d'auteur. L'utilisation des services d’Érudit (y compris la reproduction) est assujettie à sa politique d'utilisation que vous pouvez consulter en ligne.

https://apropos.erudit.org/fr/usagers/politique-dutilisation/ 


\title{
Le concept de cluster est-il soluble dans le tourisme?
}

\author{
Cécile CLERGEAU \\ Professeure des Universités en Sciences de Gestion \\ GRANEM Université d'Angers \\ UFR Ingénierie du Tourisme, du Bâtiment et des Services — ESTHUA \\ cecile.clergeau@univ-angers.fr

\section{Philippe VIOLIER} \\ UFR Ingénierie du Tourisme, du Bâtiment et des Services — ESTHUA \\ Professeur invité à l'Université de Ningbo (Chine) \\ philippe.violier@univ-angers.fr
}

\begin{abstract}
RÉSUMÉ: Dans les approches scientifiques, le concept de cluster (grappe) met en évidence, d'une part, le rôle des proximités organisationnelles, construites à partir des proximités spatiales dans la dynamique de compétitivité des destinations touristiques. D'autre part, des clusters tourisme se développent partout dans le monde. Or, le concept de cluster, tel que proposé par Porter (1998), émane de l'économie industrielle.

L'enjeu de cet article consiste donc à montrer que le simple transfert d'un outil scientifique élaboré dans le contexte industriel au management des destinations touristiques pose problème. II doit être enrichi pour comprendre les dynamiques de développement touristique local et acquérir plus d'efficacité opérationnelle. Trois axes de réflexion théorique, mais inspirés par des études de cas rencontrées dans différents travaux de recherche, sont proposés pour interroger les analyses des clusters tourisme. II convient en effet de prendre en compte trois dimensions spécifiques qui seront abordées à partir du processus de coproduction inhérent à l'activité touristique, des dynamiques de proximités singulières dans ce champ du tourisme et des caractéristiques de l'encastrement des activités touristiques dans le territoire. Enfin, les auteurs proposent un modèle de cluster plus adapté aux destinations touristiques.
\end{abstract}

Mots-clés: Tourisme, cluster (grappe), développement, local, proximité

Dans la lignée des orientations prises par la politique industrielle, le secteur du tourisme s'empare de la question des dynamiques locales de croissance et mise sur une organisation territoriale inspirée des clusters pour développer les politiques de développement des destinations. Mikael Porter (1999 : 207) définit un cluster (en français, grappe) comme "un groupe géographiquement proche d'entreprises liées entre elles et d'institutions associées relevant d'un domaine donné, entre lesquelles existent des éléments communs et des complémentarités». Ce concept, qui a servi de modèle dans le cadre de nombreuses politiques de développement industriel, en Europe comme en Amérique du Nord, fait désormais l'objet de nombreuses recherches en tourisme (Nordin, 2003; Van der Yeught, 2008; Fabry, 2008; Kim et Wicks, 2010) et inspire des expériences de développement des destinations touristiques dans le monde entier.

Le transfert de ce concept, qui émane de l'économie industrielle et de l'économie spatiale, à une activité à haut contenu en services appelle cependant une réflexion dont s'empare cet article. Les travaux de Porter (1998; 1999) concernent essentiellement l'organisation territorialisée d'une chaîne de valeur destinée à produire des biens marchands, vendus sur un marché local ou distant et consommés ultérieurement sans que le client ait participé à la conception et à la production du bien. La production d'une expérience touristique est tout autre : elle est le fait du touriste qui mobilise ressources et prestations pour vivre son expérience touristique sur le territoire où elles sont co-inventées et coproduites. Pour prendre en considération la spécificité de l'activité touristique, la recherche doit intégrer la dimension cocréative et coproductive du tourisme, et de fait enrichir le concept de cluster. Ce dernier doit en particulier tenir compte d'une dynamique de proximités singulière, le tourisme étant par nature mobilité et souvent marqué par la question de l'altérité. Il ne peut, par ailleurs, s'abstenir d'une prise en compte de la diversité des organisations socio-économiques territoriales génératrices de la dynamique de croissance, c'est-à-dire des modalités d'encastrement des activités touristiques sur les territoires considérés. 
Nous proposons ainsi de revisiter le concept de cluster pour comprendre l'organisation territoriale d'une activité de coproduction d'expérience. Trois entrées sont proposées :

- l'intensité de la coproduction et la cocréation;

- la qualité et l'intensité de la proximité organisée;

- et l'encastrement de l'activité touristique dans l'espace socio-économique.

Cette recherche est essentiellement d'ordre conceptuel. Elle trouve son origine dans une confrontation entre sciences de gestion et géographie pour comprendre les dynamiques de développement des destinations touristiques. Elle propose un enrichissement du concept de cluster permettant d'aborder une recherche qualitative multicas sur les clusters touristiques en France (recherche comparative sur Saint-Jean-de-Monts, Marne-la-Vallée, le Pays basque (GOazen)).

Nous rappelons dans une première partie les antécédents industriels de ce concept. Puis nous en proposons l'enrichissement dans une seconde partie. Nous soulignons en particulier que l'association du touriste à la cocréation des ressources et à la coproduction de l'expérience touristique exige l'intégration au moins partielle de ce dernier au sein du réseau des acteurs constitutifs du cluster. Nous interrogeons les dynamiques de proximités à l'œuvre et montrons que la question des proximités, organisée et géographique, se pose de manière singulière : l'altérité à laquelle peut être confronté le touriste appelle une intermédiation différenciée selon ses besoins et ses compétences acquises dans des expériences antérieures. Enfin, nous interrogeons la diversité des formes d'encastrement de l'activité touristique. Une dernière partie synthétise nos propositions en un modèle enrichi du concept de cluster touristique et en souligne les conséquences pour la gouvernance de ces territoires. Nous insistons en particulier sur le fait que le cluster tourisme est un lieu créé par et pour les touristes, c'est-à-dire par et pour sa confrontation au monde.

\section{Limites à l'application de la dynamique des organisations industrielles locales à la dynamique des organisations touristiques locales} Torre (2006:2) constate que «les clusters sont aujourd'hui considérés comme la base des politiques locales, voire nationales, dans de nombreux pays» et pose la question «serionsnous tous porteriens?». L'utilisation massive de ce concept ne risque-t-elle pas de le vider de son sens? Nous proposons de redécouvrir ce concept en le ramenant à des éléments simples qui le fondent mais en constituent aussi la limite pour l'analyse des activités touristiques. Nous rappelons qu'il est utilisé pour décrire une dynamique productive liée à des externalités de réseau, à la diffusion locale des connaissances et à la quasi-intégration des firmes (Torre, 2006, 2010). Si ces éléments le rendent convaincant pour analyser le tourisme, nous notons qu'il envisage l'activité économique dans une optique tout à fait industrielle : une logique d'offre et de chaîne séquencée de valeur. Nous rappelons ensuite les proximités en jeu dans cette dynamique de développement économique. Les recherches sur l'économie des proximités intéressent le tourisme en tout premier plan : si le tourisme est mobilité, il se traduit par une colocalisation temporaire et une confrontation à l'altérité qui interrogent les proximités et les intermédiations à l'œuvre. Enfin, nous soulignons la plasticité socio-économique du concept de cluster, utilisé pour étudier des organisations industrielles très diverses. Cette plasticité nous semble devoir faire l'objet de précisions dès lors que l'on analyse des territoires touristiques aux configurations socio-économiques très variées et inscrits dans des limites territoriales administratives rigides.

\section{Une dynamique productive ancrée sur un territoire} S'appuyant sur les travaux d'Alfred Marshall (1890), Becattini (1992:45) définit un district comme « une entité socio-territoriale caractérisée par la coexistence active d'une communauté ouverte d'individus et d'une population segmentée d'entreprises». Il ajoute que «[1] a population des entreprises est segmentée dans la mesure où les différentes phases du processus productif sont réparties entre ces entreprises, chacune d'entre elles se spécialisant dans l'accomplissement d'une ou plusieurs phases» (Becattini, 1992 : 45). Les territoires analysés se caractérisent ainsi par une quasi-intégration verticale des activités productives (Torre, 2006). Ils présentent des caractéristiques communes et en particulier une importante densité de PME, entretenant des relations de coopétition (Brandenburger et Nalebuff, 1995), développant des relations non marchandes portées par des réseaux sociaux étroits (Storper, 1996) et facilitées par des croyances, des valeurs et une histoire partagées. Ainsi, et comme le souligne Pecqueur (2000: 15), «le territoire est plus qu'un réseau, c'est la constitution d'un espace abstrait de coopération entre différents acteurs avec un ancrage géographique». Cet ancrage géographique facilite par ailleurs les échanges de connaissances et une émulation propices à la créativité (Nelson et Winter, 1982; Freeman et Soete, 1997; Sierra, 1997; Camagni et Maillat, 2006).

On voit l'intérêt d'un tel cadre conceptuel pour envisager l'articulation des activités touristiques sur un territoire donné, ce qui conduit certains auteurs à parler de système touristique localisé (Perret, 1992; Marcelpoil et François, 2008). Les entreprises touristiques bénéficient d'une mise en réseau, créatrice de ressources spécifiques et inimitables. Denicolai et al. (2010 : 261) soulignent ainsi que "[t]he competitive advantage of the tourism destination as a whole often relies on the overall inter-firm network configuration, more than on a few individual firm competencies». La qualité de la coordination entre les acteurs en charge de la production et de la commercialisation du séjour touristique constitue un enjeu fort de la compétitivité des destinations (Bocquet, 2008). Par ailleurs, les chercheurs en tourisme montrent l'existence de phénomènes de transfert et de diffusion de connaissances favorables à l'innovation sur les territoires touristiques (Alvarez Gonzalez et Gonzalez Moralez, 2006; Shaw et Williams, 2009).

À partir de son modèle de compétitivité du diamant, et analysant ce qui fonde l'avantage compétitif, Porter (1998) conclut lui aussi aux vertus de la proximité territoriale et de la mise en réseau des acteurs locaux. Ses recherches l'amènent à considérer l'existence de clusters, ou grappes d'activités, regroupant des entreprises, des institutions de formation et de recherche, des industries connexes, qui toutes interviennent autour ou en complémentarité d'une même chaîne de valeur. La performance des clusters vient en particulier de la 
densité du réseau des collaborations et des chaînes d'approvisionnement locales. On est ici dans une logique d'offre : c'est l'organisation territoriale de la filière qui en fonde sa performance.

L'appropriation par la recherche en tourisme, mais aussi par les politiques de développement touristique, de ce concept issu de l'analyse des organisations industrielles interroge la pertinence d'un transfert — sans amendement ni enrichissement — d'une analyse des dynamiques productives industrielles aux dynamiques productives à haut contenu en services. Ainsi, la démonstration que l'organisation territoriale d'une activité productive améliore la productivité, la compétitivité ou l'innovation repose sur l'hypothèse que les acteurs principaux de cette dynamique productive sont les producteurs, liés dans une chaîne de valeur (OECD, 1999; Kim et Wicks, 2010; Haugland et al., 2011). Or, les recherches sur l'économie et le management des services (Grönroos, 1990; Gadrey, 1992; Zeithaml et Bitner, 2003; Lovelock, 2007), dont fait partie le tourisme, montrent que l'un des acteurs majeurs de la dynamique productive est le consommateur lui-même, coproducteur du service. Il est donc raisonnable d'investiguer les conséquences de cette coproduction sur la pertinence du concept de cluster appliqué au tourisme.

\section{Une dynamique de proximités intermédiée}

Les travaux sur les dynamiques productives territoriales interrogent les logiques de proximités mises en œuvre. L’ancrage territorial des activités et la proximité géographique ne s'avèrent pas suffisants pour expliquer les dynamiques singulières de ces territoires. La coordination doit être envisagée dans une perspective «interactionniste» (Zimmermann, 2008 : 111) et le chercheur doit «reconnaitre l'idée que s'il y a proximité géographique, il n'y a pas nécessairement coordination" (Zimmermann, 2008 : 112). Rallet et Torre $(1995,2005)$ ont proposé de parler de proximité «organisée» pour évoquer une proximité qui n'est pas que géographique mais aussi relationnelle. Celle-ci peut être liée au fait que les agents économiques se reconnaissent dans des positionnements similaires ou complémentaires, dans des projets ou des organisations. On parle alors de proximité organisationnelle. Elle naît aussi du partage de codes, de valeurs ou de représentations. On parle alors de proximité institutionnelle. Cette proximité organisée est portée par des mécanismes multiples. Elle n'est pas liée à la proximité géographique, qui est neutre tant qu'elle n'est pas activée, ou peut même parfois empêcher la coopération (Tremblay et al., 2004). Ce sont plutôt les échanges, les apprentissages, les collaborations ou les comportements concurrentiels, qui vont l'encourager.

La question de la proximité se pose avec une acuité toute naturelle dès lors qu'il s'agit d'envisager les activités touristiques : les touristes préparent un déplacement, ce qui implique des relations - à distance - avec les acteurs locaux, séjournent sur le territoire, et donc interagissent à des degrés divers avec les acteurs locaux, puis s'en reviennent forts d'expériences nouvelles, de connaissances nouvelles qu'ils gardent mémoire et qui peuvent les ramener ultérieurement sur ce territoire. Ces trois temps interrogent les formes de proximités activées ou créées.
Ces dernières attestent par ailleurs de la nécessité d'une intermédiation. Dans les dynamiques d'innovation, le rôle des organismes d'intermédiation est bien connu (Clergeau et al., 2000). Il en est de même dans la formation (Bel, 2007). On peut résumer leur fonction en quatre points :

- la mise en relation, la circulation de l'information entre acteurs du territoire;

- l'animation de réseaux d'acteurs;

- la traduction, l'interprétation des propositions des acteurs permettant leur ajustement;

- et la coordination d'acteurs d'un projet commun, la définition des objectifs communs, la fédération des moyens pour les atteindre.

Dans son modèle tripolaire, Bernard Soulage (1994) mentionne la place primordiale des institutions d'intermédiation : elles constituent l'un des trois groupes d'acteurs qui activent une dynamique économique territoriale. Elles favorisent la mise en réseau, l'activation d'une proximité organisée, l'irrigation du territoire par des réseaux sociaux porteurs d'un capital social (Putman, 1995; Nahapiet et Ghoshal, 1998). Si des territoires se caractérisent par une constitution autonome de ce capital social, basé sur un ordre collectif né de l'interaction de leurs habitants, d'autres peinent à trouver dans la proximité géographique le moteur d'une dynamique collective, parce qu'elle n'est pas suffisante pour embrasser toutes les dimensions du capital social. Ces «facilitateurs de réseaux» que sont les organismes d'intermédiation constituent à ce titre des moteurs majeurs de la réussite des clusters (Bories-Azeau et al., 2008).

Le tourisme concerne un très grand nombre d'acteurs aussi divers que les pouvoirs publics, des entreprises privées, les habitants ou les touristes. Il met en jeu une confrontation à l'altérité et est fondamentalement lié à la mobilité. Il pose d'évidentes questions relatives à la mise en relation et à la coordination des acteurs, à l'animation des réseaux, à la création d'un capital social. L'organisation en cluster des territoires touristiques invite tout naturellement à interroger les dynamiques de proximités et le rôle clé des facilitateurs de réseaux, des institutions d'intermédiation, qui les activent.

\section{Une dynamique liée à la configuration socio-économique des territoires}

Courlet (2002) rappelle qu'il existe de multiples configurations socio-économiques des territoires en croissance. S'appuyant sur les travaux de Markusen (2000), on peut ainsi mettre en évidence quatre types d'agglomérations:

- le premier, le district marshallien, est composé d'un tissu dense de PME;

- le second s'articule autour d'une grande entreprise, qui n'est pas forcément originaire du système local, et entretient des relations fortes avec un tissu d'entreprises locales mais aussi avec l'extérieur;

- le troisième est marqué par la présence motrice d'une grande installation d'État (base administrative ou militaire);

- le dernier présente le réseau local le moins dense. Formé essentiellement de plateformes succursales de grandes entreprises extérieures, il est essentiellement tourné vers l'extérieur. 
Les organisations productives territorialisées ne peuvent non plus être analysées en faisant abstraction de la spécificité des activités productives concernées. Lorsqu'il parle de clusters, Porter (1998) ne spécifie pas si la dynamique économique envisagée est propre à certains types d'industries ou d'activités économiques. Pourtant, certains auteurs considèrent que les dynamiques de proximités analysées n'ont pas les mêmes enjeux selon les secteurs d'activité (MacDonald et al., 2007).

Les effets de proximité, la forme de la coordination, la nature des conventions sociales, ou les dynamiques d'innovation sont bien sûr spécifiques à chaque forme d'organisation industrielle locale. Ils génèrent des trajectoires de développement spécifiques et requièrent des modalités de gouvernance très différentes (Ehlinger et al., 2007). Les stratégies des acteurs locaux, les modalités d'interaction et de coordination, les économies d'agglomération présentent des singularités dont doit rendre compte l'analyse des proximités, mais qui ne peuvent pas être supposées identiques selon toutes les configurations locales. D'ailleurs, la coordination et les interactions économiques ne nécessitent pas forcément de colocalisation durable pour être créatrices de savoirs (Rychen et Zimmermann, 2008). La diminution des coûts de transport de biens, de personnes et d'information (NTIC) facilite des interactions et une coordination hors des territoires qui finalement peuvent se contenter d'une colocalisation épisodique pour générer une forme de proximité organisée.

L'encastrement des activités dans les territoires considérés, leur articulation avec le contexte social, semble ici encore interroger la communauté scientifique : la diversité des territoires touristiques, de leurs configurations socio-économiques, ne peut se satisfaire d'une hypothèse d'articulation harmonieuse des proximités pour étayer un modèle de cluster tourisme.

\section{Propositions pour la revisiter d'un concept permettant de rendre compte de la spécificité des activités touristiques}

Le concept de cluster fait l'objet de nombreuses recherches dans le domaine du tourisme et inspire des expériences de développement touristique dans le monde entier, depuis le Pays basque jusqu'à la Chine (Fabry, 2008; Kim et Wicks, 2010; Jackson et Murphy, 2006; Jackson, 2006). Que ce soit pour vendre une destination, accroître sa visibilité, développer sa capacité d'innovation, la nécessité d'une articulation des acteurs en des réseaux permettant la coopération appellerait une organisation territoriale de type cluster (Nordin, 2003; da Cunha et da Cunha, 2005; Alvarez Gonzalez et Gonzalez Morales, 2006). Celle-ci favoriserait la transformation de relations de compétition en relations de coopération locale, coopération dont l'impact sur la compétitivité internationale est depuis longtemps souligné. «[T]here is also an emerging shift taking in global tourism paradigm that demands greater cooperation and collaboration at the local and regional levels to ensure a quality tourism product that can compete effectively at the global level - what some authors refer to as coopetition", soulignent Crouch et al. (1999 : 139). Il s'agit bien d'insuffler de nouvelles dynamiques de développement des destinations en réponse à une concurrence accrue.
L'analyse de l'activité touristique ne doit cependant pas oublier qu'il s'agit ici de services : on ne peut faire l'économie d'une prise en compte de ce personnage essentiel qu'est le touriste, cocréateur et coproducteur de son expérience touristique. Cette dernière n'est pas le fruit d'une chaîne séquencée d'activités destinées à mettre sur le marché une offre touristique (Lanquar, 1994), mais bien le résultat d'une mobilisation sous l'impulsion du touriste, à un temps donné et sur un territoire donné, d'une constellation - et non d'une chaîne - de services destinés à coproduire une expérience. Ceci nous amène, dans la lignée des travaux de Normann et Ramirez (1993), à considérer que la destination touristique est une constellation territorialisée de valeurs créée avec et pour le touriste.

C'est sur cette base que nous proposons de revisiter le concept de cluster pour comprendre l'organisation territoriale d'une activité de coproduction d'expérience.

\section{L'intensité de la coproduction et de la cocréation}

La participation du client à la production des services fait depuis longtemps l'objet d'une littérature abondante (Eiglier et Langeard, 1987; Gadrey, 1992). Paradoxalement, les concepts de coproduction et de servuction sont peu mobilisés dans l'analyse du tourisme, et en particulier dans les approches des dynamiques territoriales. Dans la littérature sur les clusters tourisme, le touriste n'apparaît que de manière très marginale et uniquement en tant que consommateur. Or, il est toujours, certes à des degrés divers, acteur-producteur et concepteur de son expérience touristique. Pascal Cuvelier (1998) réfute ainsi l'usage du concept de consommation à propos du tourisme. Il lui substitue celui de pratique qui, selon lui, rend mieux compte de l'investissement de l'individu dans le champ touristique. D'autres chercheurs ont approfondi cette approche en analysant, à la suite de travaux d'historiens, de sociologues et de géographes (Joutard, 1986; Corbin, 1988; MacCannell, 1976; Knafou, 1992), son rôle dans l'invention des lieux et objets touristiques (Équipe MIT, 2001). Ils montrent que la ressource touristique n'existe pas en elle-même mais en relation avec les représentations des individus et des sociétés. Une illustration récente en a été apportée par la mise en tourisme du viaduc de Millau, imposée par les touristes contre les volontés de l'architecte Sir Norman Foster, des acteurs locaux et d'une partie des habitants. Les touristes rêvent leur projet touristique, le conçoivent, activent des prestataires, les coordonnent et vivent l'expérience touristique dans la destination (Violier, 2009).

Un enseignement majeur de ces analyses pour la compréhension de la dynamique productive qui anime les clusters tourisme est l'accent mis sur l'intentionnalité du touriste et sa capacité à mobiliser au profit de son projet la constellation territorialisée de valeurs. Les clusters tourisme sont animés par des relations marchandes et non marchandes que les touristes entretiennent avec les acteurs locaux, les entreprises, les institutions, les objets, mais aussi les autres touristes, pendant les moments de coprésence virtuelle - la préparation du séjour - ou effective - le séjour. Contrairement aux analyses en termes de chaîne de valeur qui l'excluent parfois 
complètement, le touriste joue au contraire un rôle déterminant face aux autres acteurs qui proposent mais ne disposent pas. En conséquence, le touriste doit être intégré au cluster.

\section{La dynamique de proximités intermédiée}

Cependant la dynamique de coproduction se heurte d'autant plus à l'étendue et à la diversité de l'espace terrestre que l'écoumène touristique, habité par les touristes, est aujourd'hui plus vaste que l'écoumène des habitants permanents. Le touriste est donc confronté à la plus grande diversité possible depuis que les moyens de transport permettent de parcourir le monde.

Le modèle de Cuvelier (1998) permet d'appréhender la diversité des «mondes» du tourisme en considérant deux continuums : la complexité/simplicité des prestations proposées par les intermédiaires et l'autonomie/hétéronomie de leur mise en ouvre. Les quatre mondes du tourisme ainsi mis en avant rendent compte de différents niveaux dans l'intensité de la coproduction de l'expérience touristique : l'autonomie renvoie à la figure d'un touriste très actif dans la cocréation et la coproduction, quand la complexité renvoie à la variété des prestations mises en œuvre et à leur articulation en une expérience touristique sur mesure.

Cette approche doit être poursuivie et complétée pour intégrer la question de l'altérité «définie comme la qualité de ce qui est autre pour un individu» (Stock, 2008 : 147). Le monde est en effet caractérisé par la diversité des milieux humains qui l'occupent et le transforment, et les touristes, lors de la réalisation de leur projet, sont confrontés à cette diversité.

Pour saisir toutes les dimensions et l'intensité de cette confrontation, nous mobilisons les analyses de Bourdieu (1979 et 1980) et de Berque (2009). Le premier montre avec le concept d'habitus que, par la socialisation, l'individu intègre des normes et valeurs sociales qui constituent des dimensions de son identité. De son côté, Berque ajoute que, contrairement à la modernité qui oppose l'humain et l'objet, le milieu est incorporé par l'individu. Il en résulte que par le tourisme, qui est un changement de place, il se retrouve confronté à des milieux dont il ne maîtrise pas les codes sur lesquels il est partiellement informé et dans lesquels son corps sera soumis à des conditions écologiques inhabituelles et éprouvantes.

Selon le degré d'altérité induit par le projet, le touriste peut être plus ou moins impliqué dans la coproduction. En effet, une altérité forte limite singulièrement ses capacités d'autonomie. Ni appartenance ni similitude ne viennent supporter une proximité entre le lieu et le touriste, et celle-ci ne reste que géographique. Il tendra alors à s'orienter vers des prestations simples ou proposées par des intermédiaires en charge de limiter les inconvénients de l'altérité, et "d'organiser» une relative proximité du touriste avec le lieu investi. Pour autant, l'intensité de la participation du touriste dépend aussi des compétences accumulées, et donc de l'apprentissage effectué, de ses expériences antérieures et de la façon dont il a capitalisé les connaissances et développé ses savoirs. Ces compétences sont acquises par l'expérience touristique, mais également en dehors, et réinvesties. Nous pensons en particulier à des capacités linguistiques acquises dans la formation (Ceriani et al., 2008). Elles se construisent dans le temps : un individu n'est pas soumis à un niveau d'altérité figé en fonction de données innées comme le donne à penser le modèle de Plog (1974). De même, la qualité de l'intermédiation requise pour accéder aux lieux n'est pas figée dans le temps : à mesure que les territoires s'ouvrent au tourisme, la qualité des intermédiations change.

Les intermédiaires constituent un ensemble qui compte les voyagistes, les agences réceptives comme les habitants du territoire de destination, voire les touristes eux-mêmes à travers les récits abondants qui fourmillent de recommandations. L'intermédiation génératrice de proximité va de la diffusion d'informations à la fédération des moyens, voire au pilotage du projet touristique.

Les intermédiaires ont alors pour mission de réinventer la réalité de la société d'accueil pour la rendre accessible et compatible avec les anticipations du touriste. Outre l'offre de facilités tangibles permettant l'accès aux lieux (management ad hoc du back office, de l'arrière-scène), ils ont alors pour fonction de créer une mise en scène de la société d'accueil, une théâtralisation de l'avant-scène du service. En ce sens, ils facilitent l'interprétation des lieux par les touristes. De leur côté, les acteurs professionnels et institutionnels du tourisme cherchent à aplanir les difficultés auxquels les touristes seront confrontés. Les infrastructures (hôtels internationaux et hôtels-clubs, moyens de transport, restaurants) sont ainsi des technologies spatiales offertes pour que l'altérité reste supportable. En effet, elles jouent le rôle d'un sas qui protège les touristes contre les agressions du milieu humain dans lequel ils sont plongés. Issus d'une société d'abondance et d'un milieu tempéré, les touristes supportent d'autant mieux l'exposition à la pauvreté et à la moiteur de la nature tropicale humide que le circuit prévoit tous les soirs, dans les hôtels aux normes internationales, un havre de paix, de bonheur et d'atmosphère climatisée.

$\mathrm{Au}$ contraire, dans un lieu qui leur est plus familier, parce qu'ils le fréquentent régulièrement ou parce que l'altérité est moins marquée, les touristes sont en mesure d'activer des ressources, de construire et produire eux-mêmes leur expérience touristique. L'intensité de la coproduction est alors plus importante. Ce tourisme de proximité constitue d'ailleurs le fait majoritaire dans tous les pays. Ainsi, la plupart des touristes sont en situation de relative familiarité, ce qui explique le recours relativement faible aux circuits tout compris, et les situations dans lesquelles la coproduction est affirmée sont les plus fréquentes. Cette proximité se traduit par le fait que les touristes disposent de compétences leur permettant d'appréhender le lieu, qu'ils entretiennent des relations avec ce lieu, voire partagent des représentations avec ses occupants (résidents ou autres touristes). Se créé ainsi une proximité organisationnelle qui peut toutefois nécessiter une forme d'intermédiation, ne serait-ce que pour animer le réseau, faire vivre et valoriser un capital culturel et social. S'il ne s'agit plus ici de créer ex nihilo et de mettre en scène une proximité entre le touriste et le lieu investi, l'intermédiation n'en est pas moins cruciale pour maintenir/développer le lien : les touristes, résidents éphémères et mobiles, développent des compétences de plus en plus grandes, leur permettant d'agrandir le cercle de ce qui leur est «proche» ou familier. 
La «clusterisation» des territoires touristiques ne peut ainsi faire l'économie d'un questionnement sur la qualité de l'intermédiation et des intermédiaires qui activent les proximités ainsi que leur évolution dans le temps. Il est ainsi pertinent, par exemple, d'interroger l'association des grands voyagistes ou groupes hôteliers, intermédiaires primordiaux des destinations touristiques lointaines, ou celle des acteurs de la vie associative locale des destinations de l'hexagone. Il semble aussi nécessaire d'examiner la force et l'influence relative de ces intermédiaires au cours du temps, à mesure que le lieu touristique se développe. La dynamique des proximités touristiques semble devoir s'appuyer sur une organisation supralocale et multiscalaire.

\section{L'encastrement du tourisme}

dans un contexte socio-économique

Comme le souligne Granovetter (1985), les acteurs économiques sont encastrés dans des contextes sociaux et institutionnels. Il en est ainsi pour la pratique touristique et les relations établies avec les autres acteurs : elles sont encastrées dans un milieu au sein duquel les acteurs sont temporairement coprésents et qui influence le déroulement du processus de coproduction. L'analyse des clusters tourisme comme organisation productive induit ainsi la prise en compte de contextes différents qui influencent son efficience.

À partir de la typologie des lieux touristiques proposée par l'Équipe MIT (2001; Stock, 2003), il nous semble possible d'esquisser un tableau qui rend compte des paramètres qui influent sur le déroulement de la coprésence et donc sur la dynamique des clusters tourisme (voir tableau 1). Ensuite dans ces lieux prévalent différentes configurations de marché. Enfin, les limites spatiales et l'étendue du cluster ne sont pas données à priori et seulement par les acteurs professionnels et institutionnels mais sont coconstruites avec les touristes.

Le tableau 1 distingue deux catégories de lieux : ceux qui sont créés par et pour le tourisme et ceux qui ont été historiquement investis. Dans les premiers, il n'existait rien ou presque avant l'irruption du tourisme qui va urbaniser le lieu. Dès lors, les acteurs du tourisme sont en situation d'imposer leurs stratégies de développement. Cette première catégorie se subdivise en comptoir ou station. Le premier sous-type est caractérisé par la fermeture, matérialisée par une clôture plus ou moins affirmée, qui isole, vis-à-vis de l'extérieur, un espace sur lequel s'exerce le contrôle d'un acteur majeur, maître du jeu. L'hôtel-club fonctionne ainsi. Inversement, la station se distingue par l'ouverture et par la mise en concurrence in situ des entreprises avec l'intervention plus ou moins marquée d'un acteur public. Toutefois, comme le tourisme constitue le moteur économique du lieu, le capital social est fondé sur le tourisme. Le pouvoir local est souvent contrôlé ou influencé par les intérêts touristiques. Les habitants, pour la plupart employés dans des entreprises du secteur, sont acquis aux intérêts du tourisme. Historiquement, l'implantation de l'espace touristique en marge du territoire induit un basculement de centralité comme à Escoublac, une commune française située dans la région des Pays de la Loire, devenue La

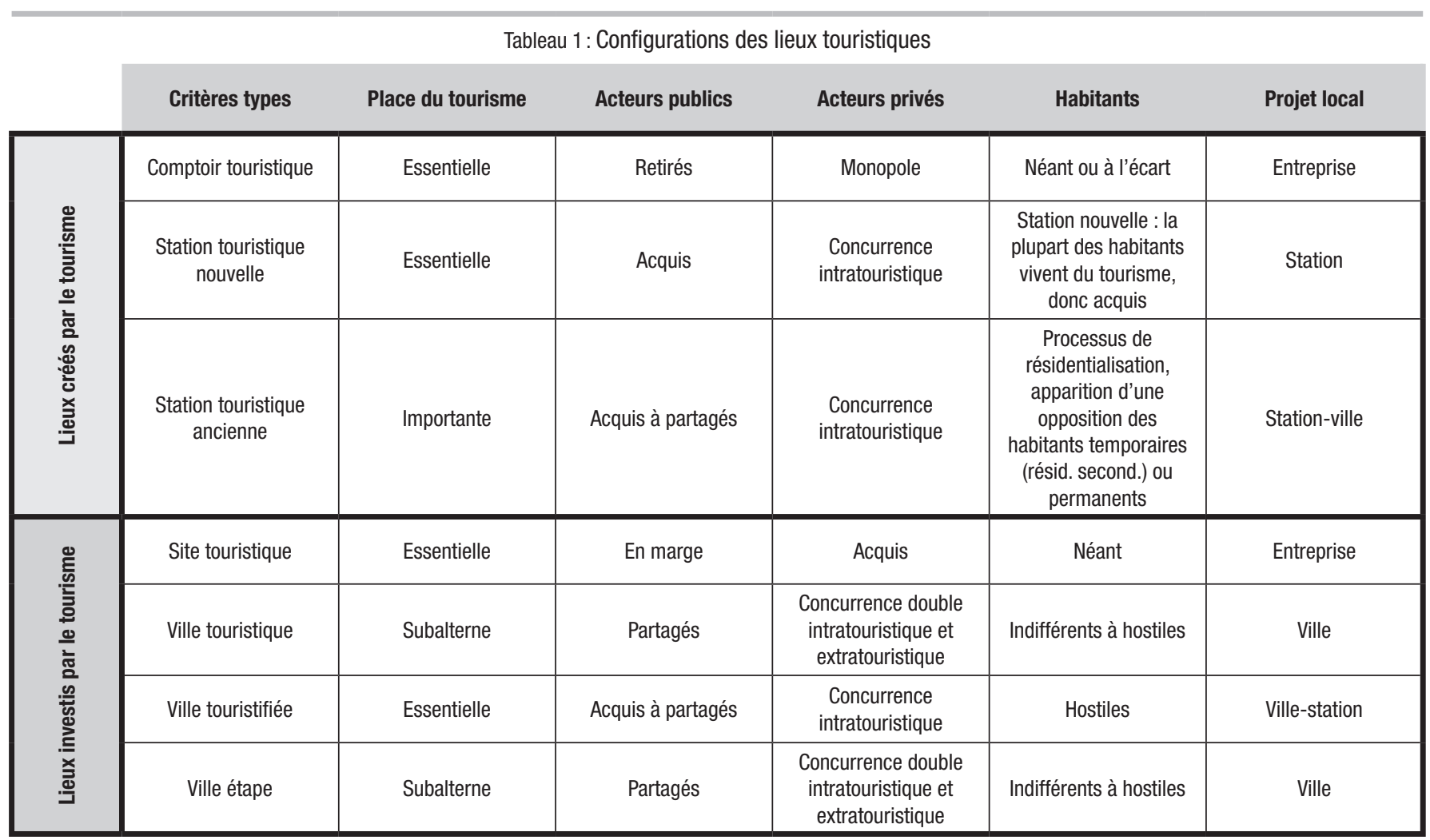

Source : équipe MIT (2001) pour la typologie. 
Baule-Escoublac (Violier, 2002). Ailleurs, le processus a abouti à la sécession du quartier touristique érigé en commune de plein droit. La construction de la proximité organisée en est facilitée en excluant ou en relativisant le pouvoir des acteurs non touristiques.

La seconde catégorie, les villes touristiques, se singularise par la diversité des fonctions. Le tourisme a en effet investi des lieux chargés d'histoire où les fonctions administratives et économiques sont plurielles. Les acteurs du tourisme y entrent en concurrence pour le partage des ressources avec d'autres intérêts. Les acteurs économiques des autres fonctions peuvent s'y opposer. Les pouvoirs publics ne sont pas d'emblée acquis au tourisme. L'inventaire même des acteurs pose problème entre ceux qui sont situés au cœur du système touristique et qui s'y reconnaissent, et d'autres, plus en marge, ou qui revendiquent d'autres appartenances comme la sphère culturelle ou sportive sans oublier les synergies avec le monde des événements d'affaires. La construction des proximités organisées se heurtent donc à davantage d'obstacles.

Au-delà, les lieux évoluent. Les comptoirs tendent à devenir des stations comme les stations se muent en villes dès lors que des fonctions non touristiques, et notamment l'accueil de résidents permanents ou semi-permanents, tendent à se développer. Le processus de résidentialisation permis par la mobilité croissante des individus change en effet la nature de la station dans laquelle les fonctions tendent à se diversifier. Cette évolution contextuelle induit une modification du poids des acteurs du tourisme qui tend à aligner la situation de coprésence sur celle des villes touristiques. Inversement, les villes touristiques connaissent également des évolutions. Certaines sont devenues des villes touristifiées lorsque l'évolution historique s'est traduite par la disparition ou l'affaiblissement de fonctions autrefois dominantes, et la montée en puissance concomitante du tourisme. Il en est ainsi de Venise, de Bruges ou de Banff. Le contexte se rapproche de celui de la station avec un capital social touristique affirmé.

Toutefois, cette clé de lecture doit être croisée avec une seconde qui met l'accent sur la typologie des acteurs en coprésence. Le fonctionnement interne du lieu tient en effet aussi aux rapports de force qui s'établissent, et notamment à la présence ou non d'établissements intégrés aux réseaux majeurs du secteur. Les systèmes touristiques localisés peuvent ainsi présenter une structure de marché de type monopolistique ou oligopolistique, marquée par la domination d'un nombre réduit de grands acteurs, ou, à l'inverse, de type totalement concurrentiel, caractérisée par un grand nombre de PME et de TPE dont la convergence difficile des stratégies nécessite une orchestration par les acteurs publics. Dans le premier cas, un ou un nombre réduit d'acteurs influence durablement la dynamique du lieu. Cette situation est plus fréquente dans les destinations majeures du tourisme, les stations huppées comme les métropoles. Inversement, dans les lieux moins renommés, le tourisme repose sur le capital régional éclaté entre de multiples entrepreneurs qui répugnent à s'associer.

Les deux précédentes approches mettent en évidence des contextes différents. Il s'agit cependant de préstructurations qui n'obèrent pas totalement la capacité d'initiative des individus. Une analyse clinique s'impose donc afin d'apprécier comment, dans un lieu précis, les acteurs parviennent à construire une proximité organisée malgré les nombreuses discontinuités qui les séparent en mobilisant les marges de manœuvres dont ils se saisissent (Friedberg, 1993).

Enfin, il découle également de la participation du touriste au processus de production que le périmètre du cluster tourisme n'est pas donné à priori par les acteurs territorialisés, ou par les institutions qui cherchent à capter au profit d'un territoire la dynamique productive, comme c'est le cas des clusters industriels. La forme spatiale du district touristique et son étendue sont incertaines. La première est donnée par l'intentionnalité du touriste. Elle dépend de la représentation que le touriste se fait de l'espace au sein duquel il déploie son projet re-créatif (Équipe MIT, 2001). Pour certains, la destination se résume à un lieu élémentaire : une station ou une ville. Pour d'autres touristes ou dans d'autres situations, la notion de destination correspond à une étendue plus vaste. En effet, le touriste demeure mobile une fois qu'il a atteint sa destination principale, si bien que l'espace du cluster peut intégrer plusieurs types de lieux élémentaires. Par exemple, un circuit de découverte de la Vallée de la Loire inclut plusieurs sites : les châteaux isolés comme Chambord, des villes touristiques (Amboise), des villes-étapes (Tours), des villages touristifiés (Chenonceau), etc. Tous ces sites pourront être visités à partir d'un lieu de séjour, une ville-étape comme Tours, bien dotée en hôtels, mais que les touristes visitent peu. L'espace du cluster résulte ainsi de la densité des liens que les touristes établissent entre des lieux en relation et avec d'autres acteurs pour la mise en œuvre de leur projet. Les professionnels et les gestionnaires peuvent bien sûr tenter d'influencer les mobilités secondes, celles qui se déploient une fois que le touriste a atteint sa destination - mobilité princeps (Piriou, 2009), mais, en dernier ressort, le touriste valide les propositions. La cartographie des clusters touristiques échappe ainsi à celle des territoires administratifs malgré les impositions territoriales intentées par les acteurs institutionnels. Ce décalage entre l'espace pratiqué par les touristes et les territoires institutionnels constitue un défi posé à la gouvernance des espaces touristiques.

Le concept de cluster, pour être pertinent dans le champ du tourisme, doit donc être, selon nous, déformé, également en raison des contextes sociaux, économiques et spatiaux dans lesquels il s'insère.

\section{Propositions pour une adaptation du concept de cluster au monde du tourisme}

Nous proposons ici d'enrichir les modèles de cluster tourisme proposés par la littérature en prenant en considération les critiques qui ont été faites. Notre modèle interroge alors la gouvernance de ces territoires.

\section{Pour une approche du cluster par les dynamiques coproductives}

En façonnant leur modèle de compétitivité des destinations touristiques, Crouch et al. (1999) s'appuient sur le modèle du diamant de Porter qui postule que la compétitivité d'une industrie donnée dépend de conditions liées à l'environnement d'affaires, à savoir les dotations factorielles, les conditions de la demande, les industries liées et supports, et les 


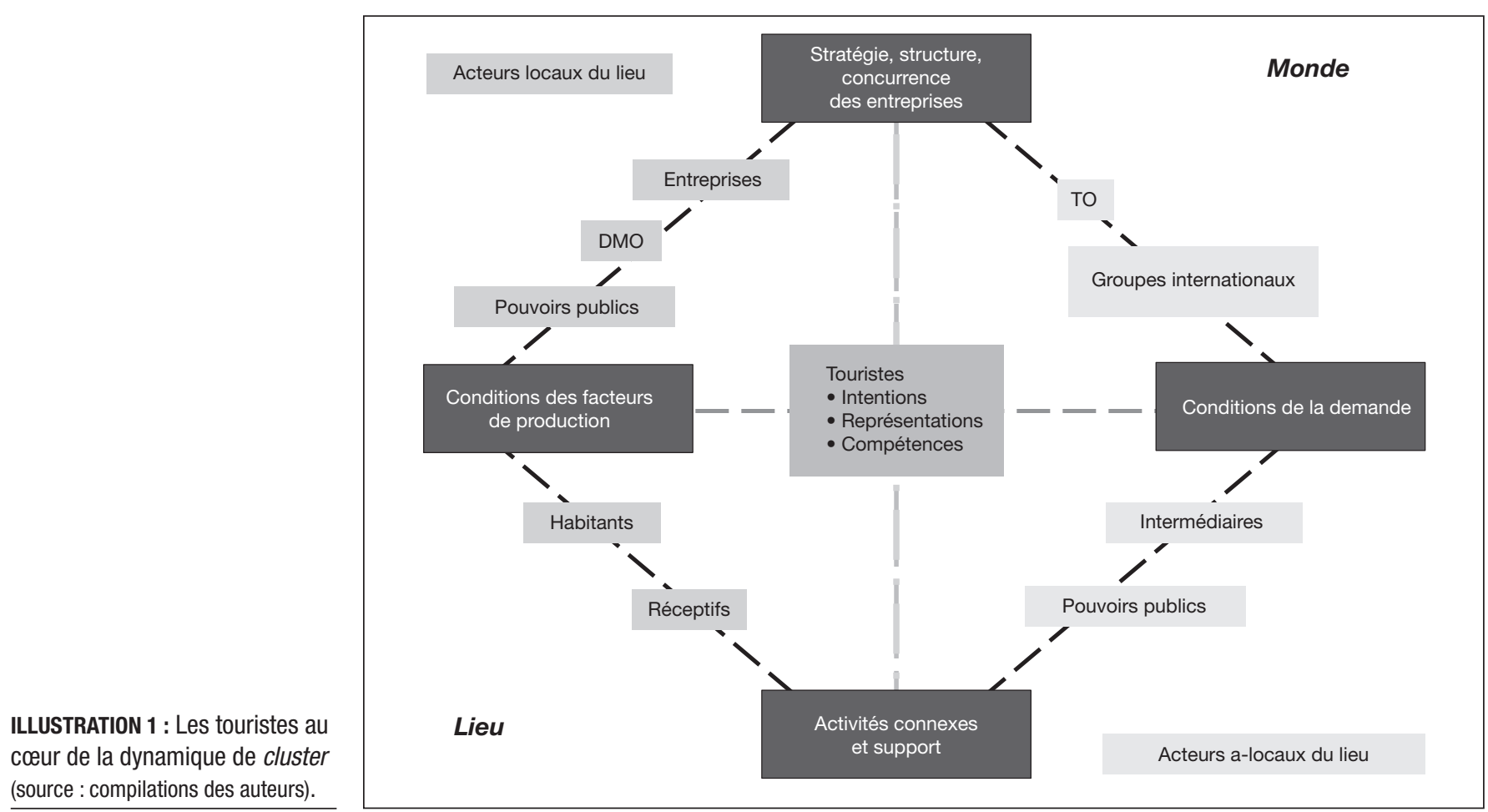

stratégies des firmes, l'intensité et la structure de la concurrence. De ce diamant est né le concept de cluster. Il est repris par De Holan et Phillips (1997), qui avaient déjà recommandé de s'en inspirer pour évaluer le tourisme dans les pays en développement. Kim et Wicks (2010) se réfèrent pareillement au diamant de Porter pour expliciter les dynamiques en jeu dans le cluster tourisme. Cependant ces modèles sont, comme nous l'avons montré, très dominés par une logique de l'offre alors même que le touriste est au cœur de la dynamique des destinations touristiques. Bien sûr, Dwyer et Kim (2003), de même que Kim et Wicks (2010), mentionnent l'importance des conditions de la demande mais celles-ci ne sont pas explicitées. Nous proposons ici d'aller au-delà de ces modèles en déplaçant l'attention d'une économie touristique de l'offre à une économie touristique de la coproduction.

Nous proposons ainsi d'enrichir l'approche des clusters en plaçant les touristes au cœur de leur dynamique (voir illustration 1).

En nous inspirant des propositions de Kim et Wicks (2010), nous différencions, dans cette présentation, acteurs et facteurs de compétitivité. Nous considérons aussi la destination touristique non comme un espace clos mais comme un espace en lien avec le monde. La destination touristique n'existe que parce qu'elle est un lieu confronté au monde et cette confrontation au monde est faite par et pour les touristes. On a montré de surcroît que ce lieu est si ouvert que ses frontières sont définies par cette confrontation au monde et aux représentations des touristes, ce qui nous amène à prendre en considération des acteurs locaux mais aussi des acteurs non locaux du lieu. Comme Kim et Wicks (2010), nous considérons que les conditions des facteurs sont bien locales et tiennent en des ressources et attractions - naturelles ou créées - liées au lieu, mais co-inventées par les touristes, cependant que les activités connexes et support de même que les conditions de la demande échappent à une vision strictement locale. Nous montrons l'importance des acteurs de l'intermédiation mais aussi celle des habitants. Enfin, cette proposition générique doit être complétée pour rendre compte de la singularité des dynamiques à l'œuvre selon que les touristes sont très éloignés de la destination et que l'altérité est forte ou, au contraire, que les touristes sont en grande proximité avec la destination. Dans le premier cas (voir illustration 2), les réseaux socio-économiques, qui supportent la dynamique du cluster, associent des acteurs non locaux et des intermédiaires nombreux. Ces derniers, même s'ils sont des entreprises productrices d'un service, se font par le même temps l'expression de la demande et créent un lien entre les touristes et les acteurs locaux. Dans le second cas, les touristes sont au cœur de dynamique de clusters, ils font le lien entre le lieu et le monde. Ils activent les prestations pour les associer en un séjour touristique, participant en cela à la production locale de séjours touristiques (voir illustration 3).

\section{De la gouvernance du cluster}

Notre modèle interroge directement la gouvernance des destinations touristiques. La cohésion des dispositifs de mise en réseau des acteurs n'est effective que si l'ensemble des parties prenantes est associé à leur gouvernance, que ces parties prenantes soient des acteurs publics locaux ou des entreprises privées internationales. Nous montrons aussi que la gouvernance des destinations touristiques doit aller au-delà d'une gouvernance de réseaux territorialisés (Ehlinger, et al., 2007), 


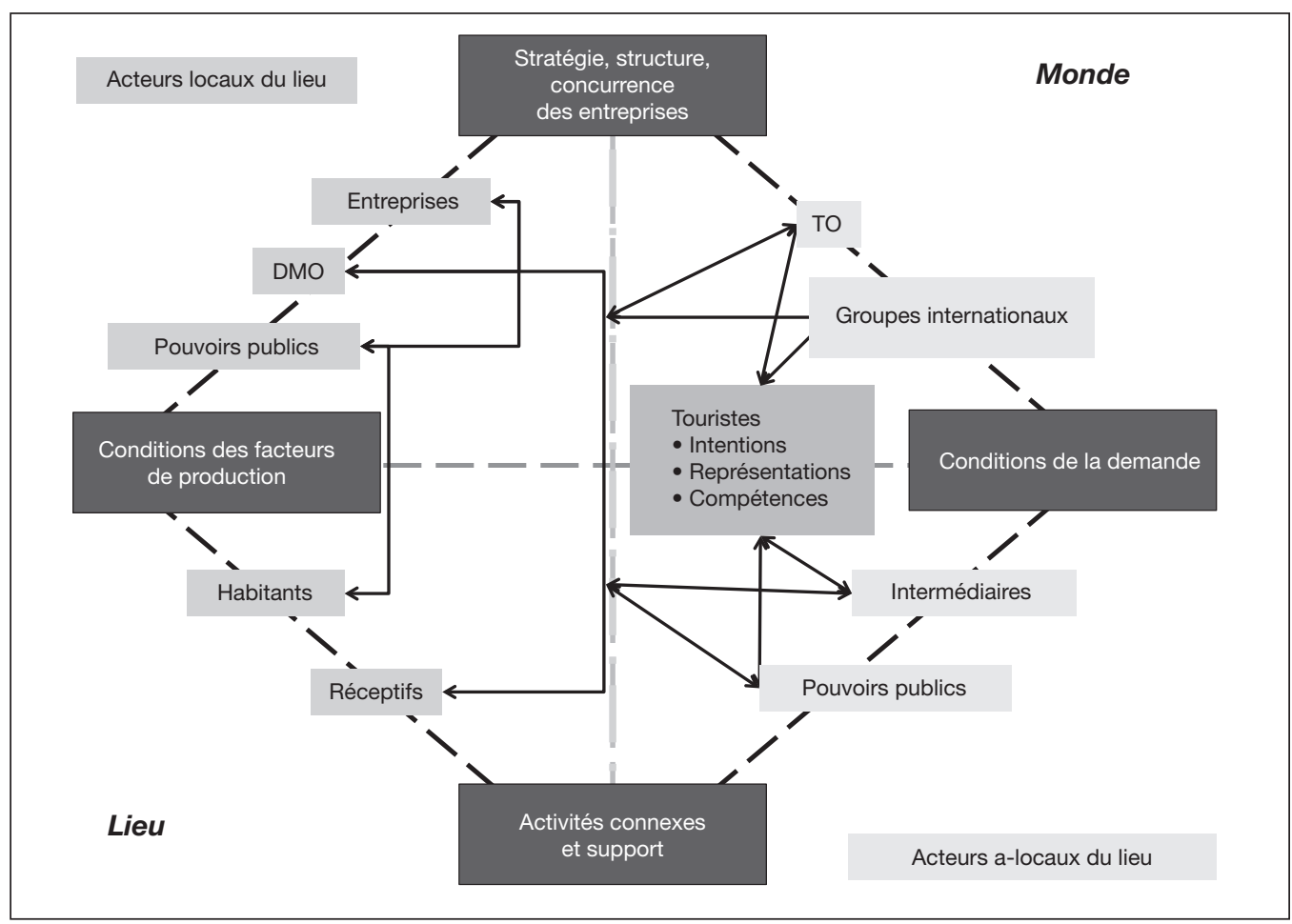

ILLUSTRATION 2 : Dynamique de cluster avec proximités intermédiées

(source : compilations des auteurs). c'est-à-dire de réseaux situés géographiquement, car les réseaux ici concernés ont la particularité d'associer des acteurs non locaux qui participent étroitement à la création locale de ressources et de richesses.

$\mathrm{Si}$, au stade de notre recherche, il est hasardeux de s'engager sur des recommandations en matière de gouvernance, notre modèle permet de soulever la question des parties prenantes. En reprenant les mots de Freeman (1984), les acteurs qui affectent ou sont affectés par l'activité du cluster tourisme constituent une mosaïque large et complexe. Notre modèle montre que ces parties prenantes dépassent largement le cadre du local. C'est le cas pour les entreprises mais aussi pour l'ensemble des acteurs du cluster, dont les habitants ou les touristes. La singularité coproductive du tourisme amène en effet à s'interroger sur l'association des touristes à la gouvernance des destinations touristiques. Habitants temporaires, les touristes sont difficilement mobilisables. Cependant, on dispose aujourd'hui de technologies fiables et puissantes permettant une forme ou une autre de participation. Il est ici important de questionner l'usage qui est fait des technologies de l'information et de la communication pour informer les touristes, animer des réseaux, développer leurs compétences ou une culture commune et les associer à la dynamique locale du cluster. Les résidents, habitants permanents, sont eux aussi parties prenantes du cluster, même s'ils ne sont pas directement concernés par le tourisme. Dans les villes touristiques, ils peuvent même être hostiles au tourisme, mais n'en restent pas moins des parties prenantes impactées par le tourisme (Clergeau et Paulus, 2012). Par ailleurs, un phénomène de déterritorialisation/reterritorialisation (Leloup et al., 2005) a considérablement accru le nombre et l'échelon des instances influentes ou décisionnaires sur le territoire touristique. La gouvernance territoriale s'incarne désormais dans des instances et des outils très concrets et divers (districts, communautés de territoires, grappes d'entreprises) qui doivent s'ouvrir à ces parties prenantes locales et non locales. Par ailleurs, et c'est ici un point important souligné par notre recherche, si les configurations socio-économiques structurent considérablement les clusters tourisme, elles forment des configurations réticulaires différenciées qui appellent des formes différenciées de gouvernance. Si l'on retient les catégories proposées par Ehlinger et al. (2007), une gouvernance territoriale associative aura du mal à s'organiser spontanément sur une station touristique nouvelle. L'acteur central qui a porté le projet de station, qu'il soit public ou privé, aura un rôle majeur dans une gouvernance qui sera plutôt de type focal. Une gouvernance territoriale, sous l'impulsion d'un acteur public ou semi-public, permettra de coordonner les acteurs d'une ville touristique. La gouvernance du cluster doit refléter sa structuration socio-économique.

\section{Conclusion}

Nous avons montré dans cet article que le concept de cluster était probablement trop étroit et devait au minimum être enrichi pour rendre compte de la dynamique des territoires touristiques. Notre modèle insiste sur le fait que la dimension coproductive du tourisme implique d'intégrer le touriste au sein du cluster. Interrogeant les proximités à l'œuvre et les phénomènes d'intermédiation, il montre qu'une dynamique territoriale exige l'association d'acteurs qui ne sont pas nécessairement coprésents et dont les cultures (professionnelles, sociétales, etc.) ne sont pas homogènes. Nous étudions enfin 
ILLUSTRATION 3 : Dynamique de cluster avec proximité (source : compilations des auteurs).

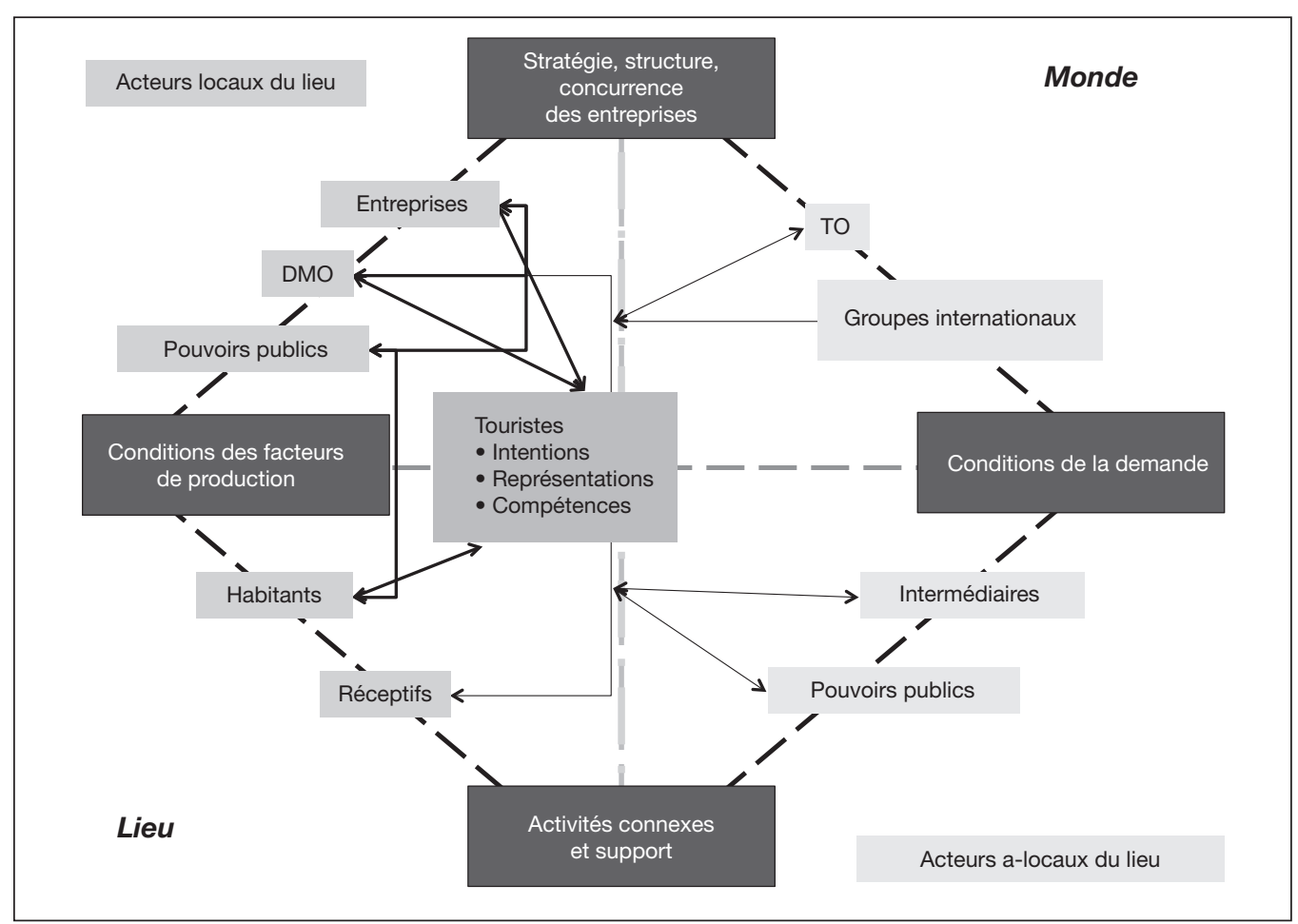

l'hétérogénéité des configurations socio-économiques et spatiales qui méritent d'être prise en considération et nous interrogent sur les bases de la cohésion des dispositifs.

Ces premiers travaux trouvent leur limite dans leur caractère conceptuel. Ils appellent une série d'études de cas de clusters tourisme. Ils ouvrent plusieurs perspectives de recherche sur les modalités d'association de ces acteurs clés, temporairement présents sur le territoire, mais rarement associés, que sont les touristes. Les technologies de l'information offrent à cet égard des outils favorisant la construction d'une proximité organisationnelle à condition, bien sûr, que des espaces leurs soient ouverts (Clergeau et Violier, 2011). Plus globalement, la gouvernance des destinations est interrogée par nos travaux, de même que les trajectoires des territoires touristiques. Les destinations changent, innovent, renouvellent leurs organisations et leurs ressources, et ce, sous l'impulsion de choix des acteurs associés, dans des processus de tri et d'erreur. La capacité d'évolution est liée à l'influence de ces parties prenantes et des jeux de conflit/coopération/négociation autour du partage des territoires qui les animent.

\section{Références}

ALVAREZ GONZALEZ, José Antonio et Dra Olga GONZALEZ MORALEZ

(2006) «L’apprentissage, l'innovation et la compétitivité dans les clusters touristiques : une étude comparée entre l'Espagne et l'Italie», Revue d'Économie Régionale et Urbaine, $\mathrm{n}^{\circ}$ 4, p. 551-574.

BECATTINI, Giacommo (1992) «Le district marshallien : une notion socio-économique», DANS BENKO, Georges et Alain LIPIETZ (sous la direction de), Les régions qui gagnent, p. 37-39. Paris : PUF.

BEL, Maïten (2007) «Formation et territoires, des approches renouvelées», Formation Emploi, no 97, p. 67-80.
BERQUE, Augustin (2009) Écoumène Introduction à l'étude des milieux humains, Paris : Belin Mappemonde. 446 p.

BOCQUET, Rachel (2008) «Proximités et coordination interfirmes : une analyse empirique de la compétitivité des stations de sport d'hiver ", Revue d'Économie Régionale et Urbaine, $\mathrm{n}^{\circ} 2$, p. 159-178.

BORIES-AZEAU, Isabelle; C. FABRE; P. FAILLENET et Anne LOUBES (2008) «La contribution de l'animateur dans la production et la mobilisation du capital social au sein d'un réseau inter firmes», DANS LECOUTRE, Marc et Pascal LIEVRE (sous la direction de), Management et réseaux sociaux, p. 279-291. Paris : Hermes Science Publishing.

BOURDIEU, Pierre (1979) La distinction : critique sociale du jugement, Paris : Les Éditions de Minuit. 670 p.

BOURDIEU, Pierre (1980) Le Sens pratique, Paris : Les Éditions de Minuit. $475 \mathrm{p}$.

BRANDENBURGER, Adam et Barry NALEBUFF (1995) «The Right Game: Use Game Theory to Shape Strategy", Harvard Business Review, juilletaoût, p. 57-71.

CAMAGNI, Roberto et Denis MAILLAT (2006) Milieux innovateurs, théorie et politiques, Paris : Economica, Anthropos. 502 p.

CERIANI, Giorgia; Vincent COËFFE; Jean-Christophe GAY; Rémy KNAFOU; Mathis STOCK et Philippe VIOLIER (2008) "Conditions géographiques de l'individu contemporain », EspacesTemps. net, textuel, 13 mars.

CLERGEAU, Cécile; Mathieu DETCHESSAHAR et Bernard QUINIO (2000) «Les politiques de développement technologique : contenu, organisation et évaluation de la fonction d'intermédiation technologique», Politiques et management public, vol. $18, \mathrm{n}^{\circ} 2$, p. 25-42.

CLERGEAU, Cécile et Krystel PAULUS (2012) Démarche RSE dans l'évènementiel : Propositions pour une analyse multidimensionnelle des parties prenantes du champévènementiel : $2^{e}$ colloque AsTRES ( 2 et 3 avril). Université de Nice, Sophia-Antipolis. 
CLERGEAU, Cécile et Philippe VIOLIER (2011) Les clusters tourisme : quels enjeux pour les systèmes d'information? Une analyse exploratoire : $X V I^{e}$ conférence de l'Association Information et Management (25 au 27 mai). Institut d'Administration des Entreprises, Saint Denis de la Réunion.

CORBIN, Alain (1988) Le Territoire du vide : L'Occident et le désir du rivage, 1750-1840, Paris : Aubier. 407 p.

COURLET, Claude (2002) «Les systèmes productifs localisés, un bilan de la littérature», Étude et recherches sur les systèmes agraires et le développement, vol. 33, p. 27-40.

CROUCH, Geoffrey Ian; John RAYMOND et Brent RICHIE (1999) «Tourism, Competitiveness, and Societal Prosperity», Journal of Business Research, vol. 44, p. 137-152.

CUVELIER, Pascal (1998) Anciennes et nouvelles formes de tourisme, Paris : L'Harmattan, Tourisme et sociétés. 238 p.

DA CUNHA, Sieglinde Kindl et João Carlos DA CUNHA (2005) «Tourism Cluster Competitiveness and Sustainability: Proposal for a Systemic Model to Measure the Impact of Tourism on Local Development », Brazilian Administration Review, juillet-décembre, vol. 4, p. 47-62.

DE HOLAN, Pablo Martin et Nelson PHILLIPS (1997) «Sun, sand, and hard currency tourism in Cuba ", Annals of Tourism Research, vol. 24, $\mathrm{n}^{\circ} 4$, p. 777-795.

DENICOLAI, Stephano; Gabriele CIOCCARELLI et Antonella ZUCCHELLA (2010) «Resource-based Local Development and Networked Core-competencies for Tourism Excellence», Tourism Management, vol. 31, p. 260-266.

DWYER, Larry et Chulwon KIM (2003) «Destination competitiveness: a model and indicators ", Current Issues in Tourism, vol. 6, $\mathrm{n}^{\circ} 5$, p. 369-413.

EHLINGER, Sylvie; Véronique PERRET et Didier CHABAUD (2007) "Quelle gouvernance pour les réseaux territorialisés d'organisations? ", Revue française de gestion, vol. 1, n 170, p. 155-171.

EIGLIER, Pierre et Eric LANGEARD (1987) Servuction, le marketing des services, Paris : McGraw-Hill. 205 p.

ÉQUIPE MIT (2001) Tourismes. Lieux communs, Paris : Belin. 319 p.

FABRY, Nathalie (2008) «Clusters de tourisme et compétitivité des acteurs, une solution pour conforter le tourisme d'affaires et événementiel parisien ", DANS LEMASSON, Jean-Pierre et P. VIOLIER (sous la direction de), Destinations et Territoires, vol 1 : Coprésence à l'œuvre, p. 55-68. Montréal : Presses de l'Université du Québec.

FREEMAN, Christopher et Luc SOETE (1997) The Economics of Industrial Innovation, London : Routledge. 470 p.

FREEMAN R., Edward (1984) Strategic management: a stakeholder approach, Boston : Pitman series in business and public policy. 275 p. FRIEDBERG, Erhard (1993) Le pouvoir et la règle, Seuil, Paris. 405 p. GADREY, Jean (1992) Économie des Services, Paris : La Découverte. 124 p. GRANOVETTER, Mark (1985) «Economic action and social structure: the problem of embeddedness ", American journal of sociology, vol. 91, $\mathrm{n}^{\circ} 3$, p. 481-510.

GRÖNROOS, Christian (1990) Service Management and Marketing, Lexington, MA : Lexington Books. 404 p.

HAUGLAND, Sven A. ; Havard NESS ; Bjorn-Ove GRØNSETH et Jarle AARSTAD (2011) «Development of tourism destinations, An integrated multilevel perspective ", Annals of Tourism Research, vol. 38, $\mathrm{n}^{\circ} 1$, p. 268-290.

JACKSON, Julie (2006) «Developing regional tourism in China: The potential for activating business clusters in a socialist market economy ", Tourism Management, vol. 27, nº 4, p. 695-706.
JACKSON, Julie et Peter MURPHY (2006) «Clusters in regional tourism An Australian case », Annals of Tourism Research, vol. 33, n 4, p. 1018-1035.

JOUTARD, Philippe (1986) L'invention du Mont-Blanc, Paris : Gallimard Juillard, Coll. «Archives ». 217 p.

KIM, Namhyun et Bruce E. WICKS (2010) «Rethinking Tourism Cluster Development Models for Global Competitiveness ", International Concil on Hotels Restaurants and Institutional Education Conference, Scholarworks@UMassAmherst, <http://scholarworks.umass.edu/refereed/CHRIE_2010/Friday/28>, consulté le 15 décembre 2012.

KNAFOU, Rémy (1992) «L’invention du tourisme», DANS BAILLY, A.; R. FERRAS et D. PUMAIN (sous la direction de), Encyclopédie de Géographie, p. 851-864. Paris : Economica.

LANQUAR, Robert (1994) L'économie du tourisme, Paris : PUF, Coll. «Que sais-je? », $4^{\mathrm{e}}$ édition. $126 \mathrm{p}$.

LELOUP, Fabienne; Laurence MOYART et Bernard PECQUEUR (2005) «La gouvernance territoriale comme nouveau mode de coordination territoriale?», Géographie, Économie et Société, no 4 vol. 7, p. 321-331.

LOVELOCK, Christopher et Jochen WIRTZ (2007) Services Marketing, $6^{\mathrm{e}}$ edition, Upper Sade River : Prentice Hall. 648 p.

MacCANNELL, Dean (1976) The tourist. A new theory of the leisure class, New York : Schocken Books. 231 p.

MACDONALD, Frank; Qihai HUANG; Dimitrios TSAGDIS et Heinz Joseph TUSELMANN (2007) «Is There Evidence to Support Porter-type Cluster Policies?» Regional Studies, vol. 41.1, p. 39-49.

MARCELPOIL, Emmanuel et Hugues FRANÇOIS (2008) «Les processus d'articulation des proximités dans les territoires touristiques ", Revue d'Économie Régionale et Urbaine, vol. 2, p. 179-191.

MARKUSEN, Ann (2000) «Des lieux-aimants dans un espace mouvant : une typologie des districts industriels ", DANS BENKO, G. et A. LIPIETZ (éd.), La richesse des régions. Pour une géographie socioéconomique, p. 85-121. Paris : PUF.

MARSHALL, Alfred (1890, rééd. 1971) Principes d'économie politique, Livre IV, traduction française de F. Sauvaire-Jourdan (1906), reproduction de la première édition française publiée à Paris : V. Giard et Brière, Paris : Gordon \& Breach. (1971). 158 p.

NAHAPIET, Janine et Sumantra GHOSHAL (1998) «Social capital, intellectual capital and the organizational advantage », Academy of Management Review, vol. 3, n 2, p. 242-266.

NELSON, Richard Rex et Sidney WINTER (1982) An Evolutionary Theory of Economic Change, Cambridge (Massachusset) : Belknap Press/ Harvard University Press. 437 p.

NORDIN, Sara (2003) Tourism, Clustering and Innovation: Paths to Economic Growth and Development, Östersund (Suède) : European Tourism Research Institute. 90 p.

NORMANN, Richard et Rafael RAMIREZ (1993) «From Value Chain to Value Constellation : Designing Interactive strategy ", Harvard Business Review, juillet-août, p. 65-77.

OECD (1999) Boosting Innovation. The cluster approach, Proceedings, France : OECD, <http://www.clusterbg.net/content/library/ EN/Boosting_Inovations_Cluster_Approach.pdf $>$, consulté le 10 octobre 2012.

PECQUEUR, Bernard (2000) Le développement local, Paris : Syros. 132 p. PERRET, Jacques (1992) «Le développement touristique local : les stations de sport d'hiver». Thèse de doctorat, Grenoble : Université Pierre Mendes France. 337 p.

TÉOROS, vol. 31, nº 2, p. 60-71 C 2012 
PIRIOU, Jérôme (2009) «Une approche régionale de la destination touristique», DANS LEMASSON, Jean-Pierre et Philippe VIOLIER (sous la direction de), Destinations et Territoires, vol. 1 : Coprésence à l'œuvre, p. 38-44. Montréal : Presses de l'Université du Québec.

PLOG, Stanley (1974) «Why Destination Areas Rise and Fall in Popularity» Cornell Hotel and Restaurant Administration Quarterly, vol. 14, $\mathrm{n}^{\circ} 4$, p. 55-58.

PORTER, Michael (1998) «Clusters and the New Economics of Competition ", Harvard Business Review, novembre-décembre, p. 77-90.

PORTER, Michael (1999) La concurrence selon Porter, Paris : Editions Village Mondial (édition originale parue sous le titre On Competition, Boston : Harvard Business School Press, 1998). 475 p.

PUTMAN, Robert D. (1995) «Bowling alone : Amercias declining social capital», Journal of Democracy, vol. 6, p. 65-78.

RALLET, Alain et André TORRE (sous la direction de) (1995) Économie Industrielle - Économie Spatiale, Paris : Economica. 473 p.

RYCHEN, Frédérik et Jean-Benoît ZIMMERMANN (2008) «Clusters in the Global Knowledge-based Economy: Knowledge Gatekeepers and Temporary Proximity», Regional Studies, vol. 42.6, p. 767-776.

SHAW, Gareth et Allan WILLIAMS (2009) «Knowledge Transfert and Management in Tourism Organisations: an Emerging Research Agenda», Tourism Management, vol. 30, nº 3, p. 325-335.

SIERRA, Christophe (1997) «Proximités, interactions technologiques et territoriales, une revue», Revue d'économie industrielle, no 82 , p. 7-38.

SOULAGE, Bernard (1994) «La place du politique dans les systèmes productifs localisés», DANS COURLET, C. et B. SOULAGE (sous la direction de), Industries, territoires et politiques publiques, p. 223-243. Paris : L'Harmattan.

STOCK, Mathis (sous la direction de) (2003) Tourisme, acteurs, lieux, enjeux, Paris : Belin Sup, Géographie. 304 p.
STOCK, Mathis (2008) «Il Mondo è mobile», DANS LÉVY, Jacques (sous la direction de), L'invention du Monde. Une géographie de la mondialisation, p. 133-158. Paris : Science-Po.

STORPER, Michael (1996) «Regional economies as relational assets», Revue d'économie régionale et urbaine, vol. 4, p. 655672.

TORRE, André (2006) «Clusters et systèmes locaux d'innovation. Un retour critique sur les hypothèses naturalistes de la transmission des connaissances à l'aide des catégories de l'économie de la proximité», Régions et Développement, $\mathrm{n}^{\circ} 24$, p. 15-44.

TORRE, André (2010) «Jalons pour une analyse des dynamiques de proximité», Revue d'Économie Régionale et Urbaine, nº 3, p. 409-437.

TORRE, André et Alain RALLET (2005) «Proximity and Localization», Regional Studies, vol. 39, $\mathrm{n}^{\circ}$ 1, p. 47-59.

TREMBLAY, Diane-Gabrielle; Juan-Luis KLEIN, Jean-Marc FONTAN et Serge ROUSSEAU (2004) «Territorial proximity and innovation: a survey of the Montreal region», Research note n²004-06 A, Canada Research Chair on the Socio-Organizational Challenges of the Knowledge Economy. 17 p. <http://www.teluq.uqam.ca/chaireecosavoir/ pdf/NRC04-06A.pdf $>$, consulté le 15 février 2012.

VAN DER YEUGHT, Corinne (2008) Favoriser le développement d'un cluster "tourisme durable" au sein d'une destination touristique : le cas des Cinq Terres (Italie) : $9^{e}$ Congrès International Francophone en Entrepreneuriat et PME (CIFEPME) (octobre), Louvain-la-neuve.

VIOLIER, Philippe (2002) «La Baule de la station au lieu de vie», Mappemonde, vol. 66, p. 20-24.

VIOLIER, Philippe (2009) Tourisme et développement local, Paris : Belin. $180 \mathrm{p}$.

ZEITHAML, Valarie et Mary Jo BITNER (2003) Services Marketing, $3^{\text {e édi- }}$ tion, Boston : McGraw-Hill Irwin. 624 p.

ZIMMERMAN, Jean-Benoît (2008) «Le territoire dans l'analyse économique», Revue Française de Gestion, n 184, p. 105-118.

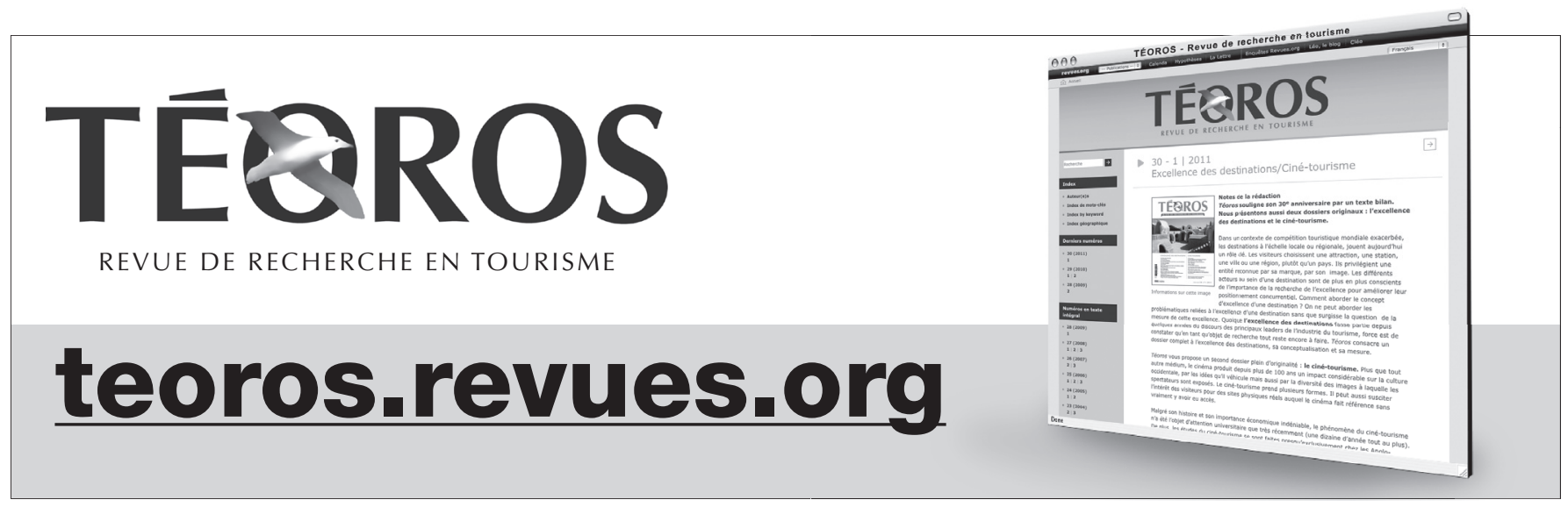

TÉOROS, vol. 31, n² 2, p. 60-71 @ 2012 